Anaesthesist 2016 $\cdot 65: 809-811$

DOI 10.1007/s00101-016-0236-6

Online publiziert: 24. Oktober 2016

๑) Springer-Verlag Berlin Heidelberg 2016

CrossMark

\title{
E. Biermann
}

Nürnberg, Deutschland

\section{"Necessitas non habet legem"?}

\section{Der Arzt im Spannungsverhältnis zwischen Berufsethos und Patientenwillen}

\section{Auch „Unvernunft" ist rechtlich geschützt}

wörtliche „Binsenweisheit“ ist rechtlich unzutreffend. Not kennt viele Gebote: Für alle - auch, aber eben nicht nur für Ärzte gilt die allgemeine Pflicht, bei Unglücksfällen, gemeiner Gefahr oder Not die erforderliche und dem Hilfspflichtigen im konkreten Fall zumutbare Hilfe zu leisten. Erst recht gilt eine Pflicht zur Hilfeleistung für die sogenannten „Garanten“, d. h. für solche Personen, die es übernommen haben, bei Gefahr und Notfällen Hilfe zu leisten, wie z. B. Polizei, Feuerwehr und eben auch Ärzte, sei es, weil sie mit der Behandlung eines Patienten eine Garantenstellung für diesen übernommen haben, sei es, weil sie sich in die Organisation des Rettungs- und Notarztdienstes eingegliedert haben. Die notallmedizinische Versorgung von Notfallpatienten, d. h. von Verletzten und Kranken, die sich in Lebensgefahr befinden und bei denen schwere gesundheitliche Schäden zu befürchten sind, wenn sie nicht unverzüglich die erforderliche medizinische Versorgung erhalten, ist detailliert in den Rettungsdienstgesetzen der Länder geregelt. Durchmustert man die Rettungsdienstgesetze, so finden sich zahlreiche „Gebote“ zur Organisation der Abläufe und zur Qualifikation der Beteiligten.

Aber auch von den allgemeinen „Geboten“ und Verboten des Straf- und $\mathrm{Zi}$ vilrechts bleiben die Ärzte in der Notfallmedizin nicht verschont. Die Notfallmedizin bewegt sich ebenso wenig wie die Medizin im Übrigen in einem „rechtsfreien Raum": Fragen der strafrechtlichen Verantwortung und der zivilrechtlichen Haftung stellen sich auch im Rahmen der Notfallmedizin.
Die Abwendung von Lebensgefahr und schweren gesundheitlichen Schäden gehört zu den ärztlichen Kernaufgaben. Die Berufs- und Therapiefreiheit des Arztes ist verfassungsrechtlich geschützt in Artikel 5 und Artikel 12 des Grundgesetzes. Doch auch wenn die Notfallversorgung als Aspekt der Daseinsvorsorge im Grundsatz Aufgabe der staatlichen Gewalt, durch die teilnehmenden Ärzte gewährleistet und verfassungsrechtlich geschützt ist, leitet sich daraus keine „therapeutische Vernunftshoheit“ des Arztes über den Patienten ab. Gemeinnutz und wie sich ein vernünftiger Patient verhalten würde und das Individualinteresse des konkreten Patienten müssen sich keinesfalls decken. Die Freiheit des ärztlichen Berufes findet ihr Pendant im Recht des Patienten auf Wahrung seiner Autonomie und Würde, verfassungsrechtlich geschützt in Artikel 1 und Artikel 2 des Grundgesetzes. Dies bedeutet: Auch dringend indizierte und im konkreten Fall mögliche und erfolgversprechende Notfalleingriffe bedürfen grundsätzlich der Einwilligung des Patienten, gegebenenfalls in Form der mutmaßlichen Einwilligung (die im Zweifel anzunehmen ist). Dennoch: Ein entgegenstehender Wille des Patienten limitiert die ärztlichen Hilfeleistungsmöglichkeiten oder schließt sie völlig aus. Das grundrechtlich garantierte Recht des Patienten auf Selbstbestimmung schützt auch seine „unvernünftige Entscheidung“ - sofern der Patient weiß, welche Konsequenzen seine medizinisch unvernünftige Entscheidung für ihn haben kann. Der
Patient hat also durchaus ein „Recht auf Unvernunft“ [1, RN 681].

Kernaufgabe des Arztes ist es, Therapieangebote $\mathrm{zu}$ definieren, doch der Patient entscheidet, ob und inwieweit er die Therapieangebote des Arztes annimmt. Auf diesen Grundsätzen beruht insbesondere auch das seit 2009 geltende „Patientenverfügungsrecht“ ( $\$$ 1901a ff. BGB). Nach \$1901a BGB kann ein einwilligungsfähiger Volljähriger für den Fall seiner Einwilligungsunfähigkeit schriftlich festlegen, ob und in welche ärztlichen Eingriffe er einwilligt oder nicht, unabhängig von Art und Stadium einer Erkrankung. Die bindende Wirkung einer solchen antizipierten Entscheidung des Patienten stellt auch die jüngste Entscheidung des Bundesgerichtshofs (Beschluss vom 06.07.2016, Az. XII ZB 61/16) zur Patientenverfügung nicht in Frage, das Gericht stellt allerdings erhöhte Anforderungen an die Konkretisierung des Gewünschten, z. B. „durch die Benennung bestimmter ärztlicher Maßnahmen oder die Bezugnahme auf ausreichend spezifizierte Krankheiten oder Behandlungssituationen“.

\section{》) Keine „therapeutische Vernunftshoheit" des Arztes über den Patienten}

Obwohl die Rechtsprechung seit Jahrzehnten, besonders deutlich bei der Rechtsprechung zur Aufklärung des Patienten, den Willen des informierten Patienten stets in den Vordergrund gerückt hatte, zeigte sie deutliche Wertungswidersprüche bei der Versorgung von Suizidanten. Während der Bundesgerichtshof früher die Auffassung 
vertrat, dass der Arzt auch gegenüber einem Suizidanten grundsätzlich zur Hilfeleistung verpflichtet blieb, erkennt die Rechtsprechung nun zunehmend an, dass die ärztliche Verpflichtung zur Lebenserhaltung und damit der Schutz des Lebens mit dem Selbstbestimmungsrecht des Patienten konkurrieren kann (weitere Nachweise bei [1, RN 664 ff.]).

Hielt die Rechtsprechung den Arzt für zur Lebensrettung verpflichtet, zumindest dann, wenn der Suizidant die Herrschaft über das Geschehen verloren hatte, z. B. bewusstlos aufgefunden wurde, und fragte allenfalls danach, ob dem Arzt ein Handeln gegen den Willen des Patienten zumutbar war, lenkt die jüngere Rechtsprechung ein, wie das erste Beispiel in dem Beitrag von Schelling zeigt. Gleichzeitig zeigt dieser Fall die "hoch emotionale" Situation, in der sich alle Beteiligten, in diesem Fall die Polizei eingeschlossen, in einer Notfallsituation wiederfinden.

\section{Reanimation bis zur Feststellung "sicherer Todeszeichen"}

Während in einem Fall "Nichtstun“ das Richtige sein kann, wenn es dem vom Patienten Gewollten entspricht, kann in einem anderen Fall das „Nicht-genügendTun" rechtlich durchaus problematisch werden. Nach den durch das Patientenrechtegesetz 2013 in das Bürgerliche Gesetzbuch eingefügten Vorschriften hat die Behandlung, also auch die notfallmedizinische, den im Zeitpunkt der Behandlung geltenden Standards zu genügen $(\$ 630 \mathrm{a}$ Abs. 2 BGB) - insbesondere, aber nicht ausschließlich, interpretiert in Leitlinien der jeweiligen Fachgebiete. Zwar differieren Art und Maß der erforderlichen Sorgfalt nach der konkreten Situation, in der sich der jeweilige Arzt befindet (Näheres bei Ulsenheimer [1, RN 101]), und insbesondere am Unfallort werden nicht dieselben Voraussetzungen gestellt werden können wie bei „wohlvorbereiteten“ Eingriffen, doch können „selbst Eil- und Notfälle ... den Standard nur dort herabsetzen, wo eine sorgfältige Organisation und Vorbereitung für sie nicht vorsorgen kann" [2]. Soweit wie möglich und recht- lich geboten, muss deshalb auch im Rahmen der (Notfall-)Medizin Vorsorge für zu erwartende Not- und Eilfälle getroffen werden. $\mathrm{Zu}$ den im Rahmen der Notfallmedizin einzuhaltenden "Standards“ gehört auch der allgemeine Grundsatz, dass Reanimationsmaßnahmen nur unterlassen bzw. eingestellt werden dürfen, soweit sichere Todeszeichen festzustellen sind. Im Zweifel ist zu reanimieren [3]. Wird von diesen Grundsätzen abgewichen, ohne dass sich dafür in der konkreten Situation des Falles eine Rechtfertigung findet, kann sich die Frage der strafrechtlichen Verantwortung und der zivilrechtlichen Haftung stellen, wie Schelling in dem zweiten von ihm geschilderten Fall plastisch darstellt. Dabei zeigt er auch die Unterschiede aufzwischen der strafrechtlichen Verantwortung mit ihren spezifischen Beweisgrundsätzen (u. a. ,,in dubio pro reo" - im Zweifel für den Angeklagten) und der zivilrechtlichen Haftung mit ihren abweichenden Beweisgrundsätzen, die nicht, wie im Strafrecht, zugunsten des Arztes, sondern häufig zulasten des Arztes und des Krankenhauses gehen (insbesondere bei Befunderhebungsfehlern, Dokumentationsmängeln und ,groben“ Behandlungsfehlern).

\section{\) Nicht immer geht es bei der rechtlichen Betrachtung von Notfallmaßnahmen um „alles oder nichts"}

Nicht immer geht es aber bei der rechtlichen Betrachtung von notfallmedizinischen Maßnahmen um „alles oder nichts", denn der Patient kann bei grundsätzlichem Wunsch, behandelt zu werden, die Hilfeleistungsmöglichkeiten des Arztes dadurch limitieren, dass er einzelnen Maßnahmen seine Einwilligung versagt. Dieses Spannungsverhältnis zwischen ärztlichen Behandlungspflichten und den Limitierungen der Behandlungsmöglichkeiten durch den Willen des Patienten macht Schelling mit dem dritten Fall am Beispiel der Notfallversorgung einer Zeugin Jehovas deutlich.

So bleibt festzuhalten, dass Not durchaus Gebote kennt, in der Medizin vor allem auch diejenigen, die zu den „Kern- geboten" des Arztes gehören und auf die sich die Ärzteschaft in den Berufsordnungen der Ärztekammern verständigt hat: So ist es Aufgabe der Ärztinnen und Ärzte,

- das Leben zu erhalten,

- die Gesundheit zu schützen und wiederherzustellen,

- Leiden zu lindern,

- Sterbenden Beistand zu leisten

( $\$ 1$ Abs. 2 (Muster-)Berufsordnung) unter einer gewissenhaften Ausübung des ärztlichen Berufes, also mit der notwendigen fachlichen Qualifikation und unter Beachtung des anerkannten Stands der medizinischen Erkenntnisse (\$ 2 Abs. 3 [Muster-]Berufsordnung). Dies steht jedoch unter dem Vorbehalt, dass jede medizinische Behandlung unter Wahrung der Menschenwürde und unter Achtung der Persönlichkeit, des Willens und der Rechte der Patientinnen und Patienten, insbesondere des Selbstbestimmungsrechtes, zu erfolgen hat; dabei ist das Recht der Patientinnen und Patienten, empfohlene Untersuchungsund Behandlungsmaßnahmen abzulehnen, zu respektieren ( $\$ 7$ Abs. 1 [Muster-] Berufsordnung).

In welchem Spannungsverhältnis der (Not-)Arzt sich dabei bewegt, macht Schelling in seinem Beitrag ,Juristische Fallstricke in der Notfallmedizin" deutlich.

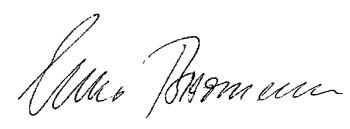

\section{E. Biermann}

\section{Korrespondenzadresse}

Dr. E. Biermann

Roritzerstr. 27, 90419 Nürnberg, Deutschland Justitiare@bda-ev.de

Interessenkonflikt. E. Biermann gibt an, dass kein Interessenkonflikt besteht. 


\section{Literatur}

1. Ulsenheimer K (2015) Arztstrafrecht in der Praxis, 5. Aufl., C.F. Müller, Heidelberg

2. Pauge B (2015) Arzthaftungsrecht - Neue Entwicklungslinien der BGH-Rechtsprechung, 13. Aufl., RN 164. RWS Verlag, Köln

3. Kern B (2010) In Laufs/Kern, Handbuch des Arztrechtes, 4. Aufl., § 17a, RN 26, 4. Kapitel Ärztlliches Berufs- und Standesrecht, \& 17a Notarzt/Rettungsdienst, RN 26. Verlag C.H. Beck, München

\section{Preise der DGAI}

\section{Pflegeförderpreis der DGAI}

Der von der Firma Heinen + Löwenstein gestiftete Pflegeförderpreis der DGAI in Höhe von 1.000,- Euro für herausragende Projekte in der Pflege zur Verbesserung der anästhesiologischen und intensivmedizinischen Versorgung steht auch zur Preisvergabe 2017 zur Verfügung.

Um den Preis können sich Einzelpersonen oder Arbeitsgruppen von Pflegekräften und Funktionskräften aus den Bereichen Anästhesie und Intensivmedizin bewerben. Die näheren Teilnahmebedingungen ergeben sich aus den Statuten des Pflegeförderpreises.

Die Statuten des Pflegeförderpreises finden Sie unter:

www.dgai.de/wissenschaftl-preise/2013-1002-09-51-36/pflegefoerderpreis

Gutachterkommission:

Prof. Dr. F. Wappler, Köln

L. Ullrich, Münster

N.N.

\section{Promotionspreis der DGA}

Die Deutsche Gesellschaft für Anästhesiologie und Intensivmedizin vergibt auch 2017 einen Preis für eine bedeutsame Dissertation auf dem Gebiet der Anästhesiologie, Intensiv-, Schmerz- oder Notfallmedizin. Die Auszeichnung ist mit 2.500 Euro dotiert.

Um den Preis können sich alle Mitglieder der Deutschen Gesellschaft für Anästhesiologie und Intensivmedizin e.V. bewerben. Die näheren Teilnahmebedingungen ergeben sich aus den u.a. Statuten des Promotionspreises. Die Statuten des Promotionspreises finden Sie unter: Anästh Intensivmed 2015;56:579580

Gutachterkommission:

Schriftführer im Präsidium der DGAI

1. Sprecher des WAKWiN

2. Sprecher des WAKWiN

Stellvertreter

Schriftführer des WAKWiN

Vertreter/in Junge Anästhesie

Berücksichtigt werden Arbeiten, die bis zum 31.01.2017(Datum des Poststempels) bei der DGAI-Geschäftsstelle, Roritzerstraße 27, 90419 Nürnberg, postalisch oder elektronisch (k.langer@dgai-ev.de) eingegangen sind. Die Arbeiten werden in dreifacher Aus- fertigung erbeten. In einem Begleitschreiben ist mitzuteilen, ob und gegebenenfalls wo die Arbeit erstmals veröffentlicht wurde. Es ist außerdem die Erklärung abzugeben, dass die Arbeit für keinen anderen Preis eingereicht worden ist. 